\title{
The clinical application of medical science research: investment and duration
}

\author{
Nao Hanaki ${ }^{1,5}$, Shintaro Sengoku ${ }^{2,5}$, Yuichi Imanaka ${ }^{3}$ and Koji Kawakami ${ }^{4,5 *}$ \\ ${ }^{1}$ Department of Healthcare Economics and Quality Management, Graduate School of Medicine, Kyoto University, Yoshida Konoe-cho, Sakyo-ku, Kyoto, 606- \\ 8501, Japan \\ ${ }^{2}$ Graduate School of Innovation Management, Tokyo Institute of Technology, W9-104, 2-12-1 Ookayama, Meguro-ku, Tokyo 152-8550, Japan \\ ${ }^{3}$ Department of Healthcare Economics and Quality Management, Graduate School of Medicine, Kyoto University, Yoshida Konoe-cho, Sakyo-ku, Kyoto, 606- \\ 8501, Japan \\ ${ }^{4}$ Department of Pharmacoepidemiology, Graduate School of Medicine and Public Health, Kyoto University, Kyoto, 606-8501, Japan \\ ${ }^{5}$ Program for Education and Research on Science and Technology in Public Sphere (STiPS), Department of Pharmacoepidemiology, Graduate School of Medicine \\ and Public Health, Kyoto University, Kyoto, 606-8501, Japan
}

\begin{abstract}
Several obstacles hamper the clinical application of basic medical scientific research. Of reports published in 6 major basic science journals between 1979 and 1983 , only 27 were translated into published randomized trials within 10 years of their initial publication. To evaluate changes in frequencies and time required for the clinical application of basic medical science research over a 10-year period, we conducted a literature search of articles published between 1989 and 1993 in eight major medical journals containing words related to clinical application in their titles or abstracts. Articles were evaluated based on whether their findings resulted in clinical application. Primary outcome was the time until translation to clinical application, which was defined as drug approval or publication of positive randomized trials. Of the 202 medical science articles identified, clinical application was eventually reported for 22. Mean time until clinical application was 22.4 years, which was longer than the 10 years previously reported $(\mathrm{p}<0.021)$. No marked differences were noted in background content between the articles that resulted in clinical application and those that did not, except for the implication of promising technologies. The prevalence of clinical application was lower and the duration until clinical application was longer than 10 years ago. Promising technologies should be carefully evaluated before study funds are allocated for clinical application.
\end{abstract}

\section{Introduction}

Numerous obstacles hamper the clinical application of medical research. Drug development, for instance, is reported to take upwards of 10 years and can cost nearly a billion United States (US) dollars [1]. Further, the cost of developing new drugs is increasing [2,3]. Promising technologies must first undergo preclinical study in the laboratory, which requires 3 to 6 years [4]. Candidate drugs must then undergo Phase 1 to 3 clinical trials in humans, which require 6 to 7 years [4]. The success rate for clinical approval is extremely low, with only 1 in every 5,000 compounds receiving approval from the Food and Drug Administration (FDA) [5]. In addition, the volume of pharmaceutical research and products decreased between 1990 and 2004 [6].

Ioannidis et al. found that, of all reports published in 6 major basic science journals between 1979 and 1983, only 27 were translated into published randomized trials within 10 years of their initial publication [7]. These authors also reported that medical studies cited more than 1000 times required 24 years to reach clinical trials [8]. Since the 1990s, the number of biotechnology-based drugs has increased [9]. Further, since the mid-1990s, a well-established process for drug discovery has been used by pharmaceutical companies [10]. Changes are anticipated in the near future regarding the medical science research field and the period required to reach clinical application. However, despite the rapid progress being made in this field, to our knowledge, no comprehensive studies in the past decade have examined the clinical application of basic medical science research. Our aim of this study is to evaluate changes in frequency and time until clinical application of medical science research over a 10 -year period.

\section{Materials and methods}

\section{Inclusion criteria}

Inclusion criteria were articles that resulted in clinical application that were published between January 1989 and December 1993 in eight major medical science journals. These eight journals consisted of the five used in the previous study [7] (Nature, Cell, Science, The Journal of Experimental Medicine and The Journal of Clinical Investigation) and three newly selected due to their high-impact factors (Nature Biotechnology and Nature Genetics derived from Nature, and Proceedings of the National Academy of Sciences of the United States of America).

As in Ioannidis et al. study, articles containing the following in their title or abstract were identified: therapy, therapies, therapeutic,

Correspondence to: Koji Kawakami, Department of Pharmacoepidemiology, School of Medicine and Public Health, Kyoto University, Yoshida Konoe-cho, Sakyo-ku, Kyoto 606-8501, Japan, Tel: +81-75-753-9469; Fax: +81-75-753-4469; E-mail: kawakami.koji.4e@kyoto-u.ac.jp

Key words: clinical application, basic medical science research, top journal

Received: June 11, 2016; Accepted: June 28, 2016; Published: June 30, 2016 
therapeutical, prevention, preventive, vaccine, vaccines, or clinical [7]. From these articles, original articles clearly stating that their findings might be clinically applicable as either or both prophylactic or therapeutic agents were selected. Eligible technologies included substances, antibodies, vaccines, gene therapies and combination therapies. In the previous study, although technical devices and other non-pharmacological interventions were included, they were not considered promising technologies [7]. These two technologies were therefore excluded from the present study and pharmaceutical agents were focused on.

Technologies still at the experimental stage (molecular, cellular, animal and early nonrandomized human trials) that did not have prior application in humans for a specific purpose were also included. Articles regarding new applications of an established technology were also included.

\section{Exclusion criteria}

The following were excluded: articles with no description of clear clinical potential in the abstract; reviews; editorials; comments; news articles; articles regarding mechanism of action, pathophysiology, diagnosis, technical devices or non-pharmacological intervention; articles regarding agricultural or veterinary applications; and articles overlapping with other articles.

\section{Screening}

PubMed (National Center for Biotechnology Information) was searched for articles that failed to meet the inclusion criteria using two steps of screening. The first step of screening was conducted based on title and abstract and the second on the full text of promising articles based on the selection rules of the reviewer. If a decision on inclusion could not be made, reviewers discussed the article in pairs and disagreements were resolved by consensus.

\section{Data extraction}

The following information was extracted from each eligible article: journal name, publication year, author information (name and institute), study design, promising technology, whether a specific technology or category of technologies was involved, anticipated application (therapeutic, preventative, or both), and disease target (cancer, nerve, cardiology, metabolism/endocrine secretion, or other). We also extracted information regarding industry involvement, defined as reported author affiliation, financial support unconnected to an author (industry funding received when no authors belonged to industry), provision of technology unconnected to an author (technology provided by industry when no authors belonged to industry), or none reported. In Ioannidis et al. study, the anticipated application was categorized as therapy, preventative, vaccine, or both therapy and prevention [7]. In the present study, given that some vaccines have a role in both therapy and prevention, we used categories of therapy, prevention, and both therapy and prevention.

\section{Identification of human studies and determination of clinical use and development status}

Literature searches of PubMed and Integrity (Thomson Reuters) for papers published through December 2014 were conducted with consideration of all alternative names of experimental pharmaceutical agents, including drug and chemical substances. To identify human randomized controlled studies, only "Human Studies" and "Randomized Controlled Trial" or "Clinical Trial" were considered.
Searches included articles that cited each eligible article to prevent potential oversight.

The following information was extracted for each eligible experimental pharmaceutical agent: development status (stage of clinical studies and general name of pharmaceutical agent) and first positive results for a randomized controlled trial (journal name, publication year, institute, industry involvement, and anticipated application). Searches were conducted in December 2014.

\section{Outcomes}

The primary outcome of interest was the time until translation to clinical application, which was defined as drug approval or publication of positive findings in randomized trials. If both outcomes occurred, the first to occur was defined as the primary outcome.

\section{Statistical analysis}

Kaplan-Meier curves were constructed for the time from publication to clinical application. Log rank test was conducted for comparison with the previous study [7]. Chi-square test was also conducted to compare backgrounds between articles resulting in clinical application and those not. Data were analyzed using JMP Pro 11 software (SAS Institute Inc., Cary, NC, USA). Significance level was less than 0.05 .

\section{Results}

Of the 38,655 articles published from 1989 to 1993 in 8 major medical science journals, 1169 were selected based on key words. This number was then reduced to 202 after examination of abstracts and original articles (Figure 1).

None of these 202 articles were published in Nature Biotechnology, and only 1 was published in Cell. However, more articles were published in these journals in the latter half of the period covered in the previous study (from 1991 to 1993). More than half of the articles selected were animal studies, and approximately $70 \%$ focused on a specific technology. In the categories of promising technology, "Proteins, peptides, and amino acids" was the most extracted category, followed by "Substances" (Table 1).

\section{Translation to clinical application}

Of the 202 articles selected, 22 were eventually translated into clinical application, of which 21 were publications of positive randomized trials, 6 were drug approvals, and 5 were both. No marked differences were noted between articles that resulted in clinical application and those that did not with respect to background content, industry involvement, and categories of promising technologies. Significant differences were only noted in implications of promising technologies, with articles featuring implications for both therapy and prevention tending to advance to clinical application over those with no such implications (Table 2).

A total of $10.1 \%$ of examined articles were therefore eventually translated to clinical application, compared with the 19.8\% (20 of 101 articles) reported in the previous study.

\section{Period until clinical application}

Mean period from publication until clinical application was 22.4 years in our study and 18.7 years in the previous study (Figure 2), a difference found to be significant using the log rank test $(\mathrm{p}<0.021)$. 


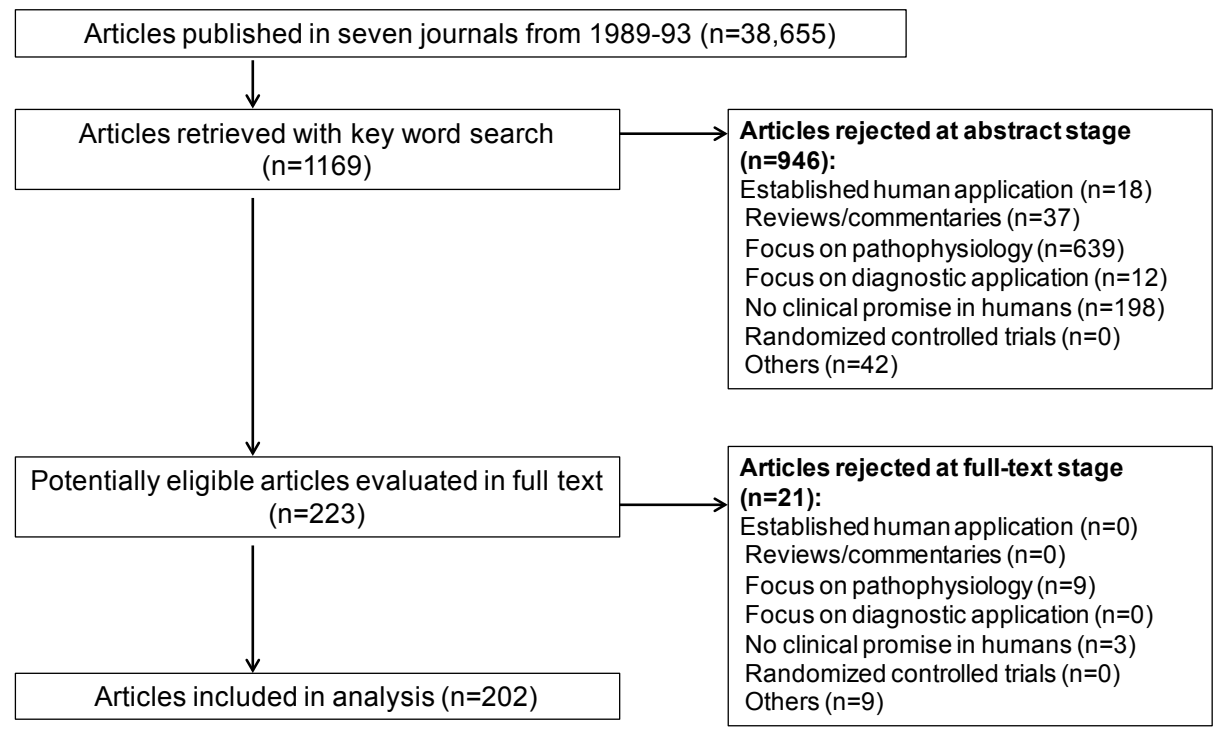

Figure 1. Selection of publications with promising therapeutic or preventive applications.

Figure 1 shows a selection process of publications with promising therapeutic or preventive applications. Of the 38,655 articles published from 1989 to 1993 in 8 major medical science journals, 1169 were selected based on key words. This number was then reduced to 202 after examination of abstracts and original articles.

Table 1. Characteristics of eligible publications.

\begin{tabular}{|c|c|}
\hline & Number $(n=202)$ \\
\hline \multicolumn{2}{|l|}{ Journal } \\
\hline Nature & 14 \\
\hline Cell & 1 \\
\hline Science & 26 \\
\hline Journal of Experimental Medicine & 18 \\
\hline Journal of Clinical Investigation & 45 \\
\hline Proceedings of the National Academy of Science & 92 \\
\hline Nature Genetics & 6 \\
\hline Nature Biotechnology & 0 \\
\hline \multicolumn{2}{|l|}{ Year } \\
\hline 1989 & 35 \\
\hline 1990 & 38 \\
\hline 1991 & 45 \\
\hline 1992 & 43 \\
\hline 1993 & 41 \\
\hline \multicolumn{2}{|l|}{ Industry involvement } \\
\hline Author affiliation & 68 \\
\hline Financial support without author & 11 \\
\hline Provision of technology without author & 7 \\
\hline None reported & 116 \\
\hline \multicolumn{2}{|l|}{ Type of study } \\
\hline Animal & 122 \\
\hline Cellular & 64 \\
\hline Human & 13 \\
\hline Molecular & 3 \\
\hline \multicolumn{2}{|l|}{ Category of promising technology } \\
\hline Antibody & 15 \\
\hline Cell & 1 \\
\hline Protein/peptide/amino acid & 60 \\
\hline Substance & 49 \\
\hline Vaccine & 34 \\
\hline Vector/gene/nucleic acid & 43 \\
\hline \multicolumn{2}{|l|}{ Type of candidate technology } \\
\hline Specific technology & 138 \\
\hline Categories of technology & 64 \\
\hline
\end{tabular}

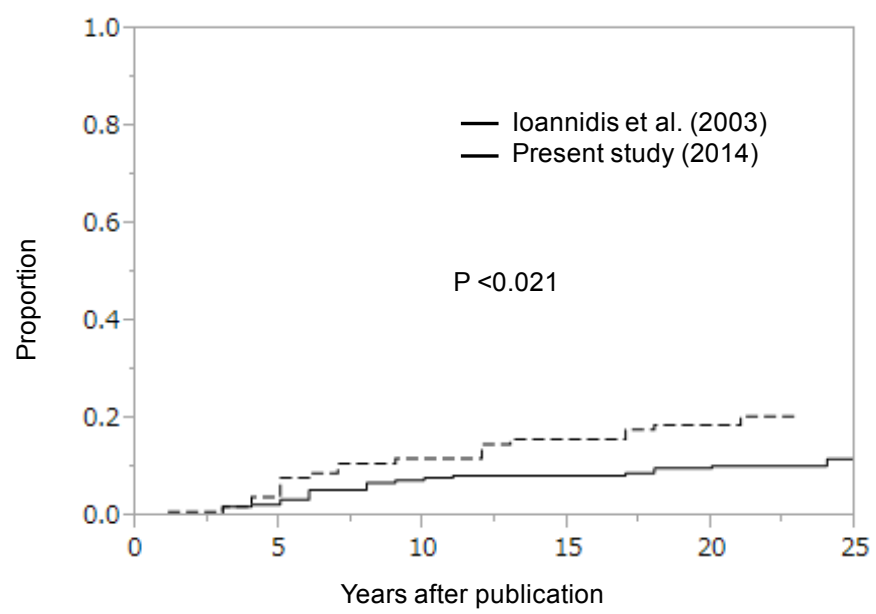

Figure 2. Proportion of candidate technologies translated to at least one positive randomized controlled trial or approval following initial publication.

Figure 2 shows Kaplan-Meier curves of proportion of candidate technologies translated to at least one positive randomized controlled trial or approval following initial publication. The dash line is the result of Ioannidis et al.'s study. The solid line is the result of present study. Mean period from publication until clinical application was 22.4 years in our study and 18.7 years in the previous study.

\section{Discussion}

Of the 202 articles identified through our literature search, only 22 were eventually translated into clinical application, with the mean period of time required until clinical application being 22.4 years-a duration significantly longer than that reported in the previous study. No marked differences were noted in background content between the articles that resulted in clinical application and those that did not, except for the implication of promising technologies. To our knowledge, this is the first comprehensive study to compare the clinical application of basic medical science research in the last decade.

Regarding the relationship between involvement of a pharmaceutical company and eventual clinical application, while some studies reported that funding by the pharmaceutical company was associated with positive findings in clinical trials [11-13], others 
Table 2. Factors associated with clinical application.

\begin{tabular}{|c|c|c|c|}
\hline & $\begin{array}{l}\text { Positive RCT or } \\
\text { Licensed/approved }\end{array}$ & Others & \\
\hline Variable & $\%(n=22)$ & $\%(n=180)$ & \\
\hline Industry Involvement & & & $\mathrm{p}=0.98$ \\
\hline Author affiliation & $36 \%(8)$ & $33 \%(60)$ & \\
\hline Financial support without author & $5 \%(1)$ & $6 \%(10)$ & \\
\hline Provision of technology without author & $57 \%(1)$ & $58 \%(6)$ & \\
\hline None reported & $3 \%(12)$ & $3 \%(104)$ & \\
\hline Type of study & & & $\mathrm{p}=0.31$ \\
\hline Animal & $45 \%(10)$ & $62 \%(112)$ & \\
\hline Cellular & $36 \%(8)$ & $31 \%(56)$ & \\
\hline Human & $14 \%(3)$ & $6 \%(10)$ & \\
\hline Molecular & $5 \%(1)$ & $1 \%(2)$ & \\
\hline Promising technology & & & $\mathrm{p}=0.27$ \\
\hline Antibody & $5 \%(1)$ & $8 \%(14)$ & \\
\hline Cell & $0 \%(0)$ & $1 \%(1)$ & \\
\hline Protein/peptide/amino acid & $45 \%(10)$ & $28 \%(50)$ & \\
\hline Substance & $32 \%(7)$ & $23 \%(42)$ & \\
\hline Vaccine & $14 \%(3)$ & $17 \%(31)$ & \\
\hline Vector/gene/nucleic acid & $5 \%(1)$ & $23 \%(42)$ & \\
\hline Type of promising technology & & & $\mathrm{p}=0.32$ \\
\hline Specific technology & $59 \%(13)$ & $69 \%(125)$ & \\
\hline Categories of technology & $41 \%(9)$ & $31 \%(55)$ & \\
\hline Implication & & & $\mathrm{p}=0.02$ \\
\hline Therapeutic & $73 \%(16)$ & $80 \%(143)$ & \\
\hline Preventive & $14 \%(3)$ & $18 \%(32)$ & \\
\hline Therapeutic and preventive & $14 \%(3)$ & $2 \%(4)$ & \\
\hline Target of potential application & & & $\mathrm{p}=0.73$ \\
\hline 1: Cancer & $9 \%(2)$ & $17 \%(30)$ & \\
\hline 2: Nerve & $14 \%(3)$ & $11 \%(20)$ & \\
\hline 3: Cardiology & $0 \%(0)$ & $3 \%(6)$ & \\
\hline 4: Metabolism/endocrine secretion & $9 \%(2)$ & $6 \%(10)$ & \\
\hline 5: Others & $68 \%(15)$ & $63 \%(114)$ & \\
\hline
\end{tabular}

reported no relationship [14,15]. Two articles included implications for their technology in both therapy and prevention for infectious disease $[16,17]$, while one included implications for memory retention [18]. Research for pharmaceutical agents for both therapy and prevention might receive more funding than those for only therapy or prevention, possibly explaining why articles with implications for both tended to advance to clinical application.

In the present study, the proportion of reports reaching clinical application was lower and the period of time between publication and application longer than reported in the previous study. It was reported that the time until translation to clinical application varied according to the definition of clinical application [19]. However, our result was the same for decreasing of success rate of researcher who were funded by NIH R01 research grant [20]. This discrepancy might be due to emergence new categories of promising technologies. For example, in the previous study, "Substances" covered 70\% of promising technologies in eligible basic science articles, followed by "Vaccines". In the present study, however, "Substances" covered only $24 \%$, with an increasing percentage of new categories such as "proteins, peptides, and amino acids" and "vectors, genes, and nucleic acids". The proportion of "Substances" that advanced to clinical trials has remained consistent for the last 50 years [21]. These new categories might complicate the evaluation of subsequent clinical application.
Follow-up was 20 to 24 years in the previous study and 22 to 26 years in the present study. However, despite the longer follow-up, the proportion of reports reaching clinical application was lower and the time until application longer in the present study than in that conducted in 2003. The effect of these different follow-up periods might therefore be negligible. The number of patients in clinical trials has increased in recent years [22], and the number of clinical trials required for clinical approval is also increasing, along with the complexity of emerging treatments [22]. These problems might account for the prolonged time to clinical application.

A potential limitation to the present study warrants mention. Given that most research is published in English and our group is most familiar with Japanese, we only searched for articles written in these languages. Articles on randomized clinical trials published in languages other than English or Japanese journals may therefore have been missed. However, given that the majority of randomized clinical trials for promising pharmaceutical agents appear to have been published in English, this limitation may be negligible.

Zanamivir [23] and Abatacept [24] are blockbuster drugs developed through application of medical science research examined in the present study. However, research which led to the development of Sitagliptin, another blockbuster drug, was first published in The Journal of Medical Chemistry [25], which was not included in the present study. Basic medical science research resulting in clinical application might not be published exclusively in well-reputed journals.

\section{Conclusions}

We demonstrated that even in research published in major medical science journals, the prevalence of clinical application was lower and required more time than that reported 10 years prior. This finding suggests that research published in well-reputed journals does not always result in clinical application. Promising technologies should be carefully evaluated before study funds are allocated for the purpose of clinical application.

\section{Authorship and contributors}

$\mathrm{NH}$ has had the main responsibility for calculating statistics and writing the paper. $\mathrm{KK}$ is the principal investigator for the project, has planned the present paper jointly with $\mathrm{NH}$, and has actively taken part in revising the paper. SS and YI have taken part in planning and analyzing data and revising paper.

\section{Disclosure of potential conflicts of interest}

The authors have no conflicts of interest directly relevant to the content of this article and there has been no significant financial support for this work that could have influenced its outcome.

\section{References}

1. Morgan S, Grootendorst P, Lexchin J, Cunningham C, Greyson D (2011) The cost of drug development: a systematic review. Health Policy100: 4-17. [Crossref]

2. DiMasi JA, Hansen RW, Grabowski HG (2003) The price of innovation: new estimates of drug development costs. J Health Econ22: 151-185. [Crossref]

3. Paul SM, Mytelka DS, Dunwiddie CT, Persinger CC, Munos BH, et al. (2010) How to improve R\&D productivity: the pharmaceutical industry's grand challenge. Nat Rev Drug Discov9: 203-214. [Crossref]

4. PhRMA Profile 2008 Pharmaceutical Industry, http://www.phrma-jp.org/archives/pdf/ profile/2008_20Profile.pdf\#search='Pharmaceutical+Industry+Profile+2008'.

5. Tufts CenterFor The Study Of Drug Development Backgrounder: How New Drugs 
Move Through the Development and Approval Process, http://csdd.tufts.edu/files/ uploads/how_new_drugs_move.pdf.

6. Pammolli F, Magazzini L, Riccaboni M (2011) The productivity crisis in pharmaceutical R\&D. Nat Rev Drug Discov 10: 428-438. [Crossref]

7. Contopoulos-Ioannidis DG, Ntzani E, Ioannidis JP (2003) Translation of highly promising basic science research into clinical applications. Am J Med114: 477-484. [Crossref]

8. Contopoulos-Ioannidis DG, Alexiou GA, Gouvias TC, Ioannidis JP (2008) Medicine. Life cycle of translational research for medical interventions. Science 321: 1298-1299. [Crossref]

9. Efpia Industry in Figures, http://www.efpia.eu/uploads/Modules/Documents/figures2007-update.pdf

10. Mayr LM, Fuerst P (2008) The future of high-throughput screening. J Biomol Screen 13: 443-448. [Crossref]

11. Davidson RA (1986) Source of funding and outcome of clinical trials. J Gen Intern Med 1: 155-158. [Crossref]

12. Bekelman JE, Li Y, Gross CP (2003) Scope and impact of financial conflicts of interest in biomedical research: a systematic review. JAMA 289: 454-465. [Crossref]

13. Sismondo S (2008) Pharmaceutical company funding and its consequences: a qualitative systematic review. ContempClin Trials29: 109-113. [Crossref]

14. Brown A, Kraft D, Schmitz SM, Sharpless V, Martin C, et al. (2006) Association of industry sponsorship to published outcomes in gastrointestinal clinical research. ClinGastroenterolHepatol4: 1445-1451. [Crossref]

15. Barden J, Derry S, McQuay HJ, Moore RA (2006) Bias from industry trial funding? A framework, a suggested approach, and a negative result. Pain 121: 207-218. [Crossref]

16. Kajigaya S, Fujii H, Field A, Anderson S, Rosenfeld S, et al. (1991) Self-assembled
B19 parvovirus capsids, produced in a baculovirus system, are antigenically and immunogenically similar to native virions. ProcNatlAcadSci USA88: 4646-4650. [Crossref]

17. Jaffe HA, Danel C, Longenecker G, Metzger M, Setoguchi Y, et al. (1992) Adenovirusmediated in vivo gene transfer and expression in normal rat liver. Nat Genet 1: 372-378. [Crossref]

18. Flood JF, Morley JE, Roberts E (1992) Memory-enhancing effects in male mice of pregnenolone and steroids metabolically derived from it. ProcNatlAcadSci U S A89: 1567-1571. [Crossref]

19. Morris ZS, Wooding S, Grant $\mathbf{J}$ (2011) The answer is 17 years, what is the question: understanding time lags in translational research. $J$ R Soc Med 104: 510-520. [Crossref]

20. Fang FC, Casadevall A (2010) Lost in translation--basic science in the era of translational research. Infect Immun 78: 563-566. [Crossref]

21. DiMasi JA, Feldman L, Seckler A, Wilson A (2010) Trends in risks associated with new drug development: success rates for investigational drugs. ClinPharmacolTher 87 : 272-277. [Crossref]

22. Scannell JW, Blanckley A, Boldon H, Warrington B (2012) Diagnosing the decline in pharmaceutical R\&D efficiency. Nat Rev Drug Discov11: 191-200. [Crossref]

23. vonItzstein M, Wu WY, Kok GB, Pegg MS, Dyason JC, et al. (1993) Rational design of potent sialidase-based inhibitors of influenza virus replication. Nature363: 418-423. [Crossref]

24. Lenschow DJ, Zeng Y, Thistlethwaite JR, Montag A, Brady W, et al. (1992) Long-term survival of xenogeneic pancreatic islet grafts induced by CTLA4lg. Science 257: 789792. [Crossref]

25. Kim D, Wang L, Beconi M, Eiermann GJ, Fisher MH, et al. (2005) (2R)-4-oxo-4 [3-(trifluoromethyl)-5,6-dihydro[1,2,4]triazolo[4,3-a]pyrazin-7(8H)-yl]-1-(2,4,5trifluorophenyl)butan-2-amine: a potent, orally active dipeptidyl peptidase IV inhibitor for the treatment of type 2 diabetes. $J$ Med Chem 48: 141-151. [Crossref]

Copyright: (C2016 Hanaki N. This is an open-access article distributed under the terms of the Creative Commons Attribution License, which permits unrestricted use, distribution, and reproduction in any medium, provided the original author and source are credited. 